\title{
II.i).Translational research
}

101

\section{EFFECT OF HIPEC ON IMMUNE MICRO ENVIRONMENT}

\section{M.D. Ray}

\section{Department of Surgical Oncology, AlIMS - Delhi (India)}

\section{Objectives}

Introduction: Patients of peritoneal Surface malignancies are usually succumbed because of metastatic disease and micro metastasis. HIPEC is the Promising novel treatment modality providing long term survival benefit for the selected patients with peritoneal carcinomatosis.

\section{Methods}

Rationale:

In vast majority of the cases the disease is confined to the peritoneal cavity for a long time. Even in recurrent cases, disease remains confined to peritoneal cavity only. Intra peritoneal administration of chemotherapy results in high peritoneal to plasma ratios for pick concentration of chemotherapeutic drugs.

\section{Results}

Mechanisms of immune activation:

Surface molecular expression- Heated tumour cells increase the surface expression of MHC class I, making the tumour cells more sensitive to lysis by CD8+T (T cytotoxic) cells. Expression of HSPs- Heated tumour cells release Heat shock protein (HSPs) which activate Natural killer (NK) cells and Antigen presenting cells (APCs), cross present the antigen to CD8+T cells. Exosome production- Heated tumour cells release exosomes, containing potential antigens, and Antigen presenting cells, cross present the antigen to CD8+ T cells. Direct effect on immune cells- NK cells, CD8+ T cells and Dendritic cells (DC), Tumour vasculature- Increases the permeability of tumour vasculature. Changed vasculature within the tumour may help immune cells mobilization

\section{Conclusion}

Conclusion:

HIPEC elicit anti-tumour immune responses by enabling tumour cells to stimulate the immune system through increased surface expression of MHC class I, release of HSPs, Exosomes, directly activating intratumoural immune cells and Improving immune-cell trafficking. 
102

PROLONGED HYPERTHERMIC INTRAPERITONEAL CHEMOTHERAPY (HIPEC) CAN TRIGGER A SYSTEMIC INFLAMMATORY RESPONSE. A STUDY IN HUMANS

\author{
L. Roth ${ }^{1}$, D. Eshmuminov ${ }^{1}$, F. Laminger ${ }^{2}$, C. Koppitsch ${ }^{2}$, M. Schneider ${ }^{1}$, T. Reding Graf ${ }^{1}$, K. \\ Slankamenac ${ }^{1}$, A. Gupta ${ }^{1}$, F. Kober ${ }^{2}$, S. Roka ${ }^{2}$, P. Gertsch ${ }^{1}$, K. Lehmann ${ }^{1}$ \\ ${ }^{1}$ University Hospital Zurich - Zurich (Switzerland), ${ }^{2}$ Center for Peritoneal Carcinomatosis, Hanusch \\ Krankenhaus - Vienna (Austria)
}

\title{
Objectives
}

CRS/HIPEC has become a treatment of choice for many patients with peritoneal metastasis. However, the pathophysiology behind HIPEC is only poorly understood, and there is an ongoing discussion which protocol to use for HIPEC: a mitomycin $C$ based protocol currently preferred by many surgeons in the US, or the oxaliplatin based protocol developed by FRENCH groups. Here, we describe for the first-time dramatic changes in the postoperative systemic inflammatory reaction, highly different among treatment protocols.

\section{Methods}

HIPEC was performed with the US protocol $\left(90 \mathrm{~min}, 42^{\circ} \mathrm{C}\right.$ with mitomycin $\mathrm{C} /$ doxorubicin) or the $\mathrm{FRENCH}$ protocol (30 $\mathrm{min}, 43^{\circ} \mathrm{C}$ with oxaliplatin). Serial blood samples were assessed for white blood cells (WBC), C-reactive protein (CRP), procalcitonin (PCT), Interleukin-6 (IL-6), pancreatic stone protein (PSP), and bacterial components (16s rDNA). The study was approved by the local ethics committee (KEK 2015-0529) and registered at clinicaltirals.gov (NCT02741167).

\section{Results}

Overall, $n=248$ patients were included. In patients without postoperative complications a secondary peak for CRP $(p=0.037)$, and PSP $(p=0.011)$ was observed after US-HIPEC, in contrast to FRENCH-HIPEC. Postoperative serum bacterial 16srDNA level were 2.1 times higher $(95 \% \mathrm{Cl} 0.646-3.032, p=0.015)$ in patients after US-HIPEC. In patients after US-HIPEC, CRP levels lacked specificity, in contrast to PCT $(80.6 \%$ vs. $15.5 \%)$, and leukocyte reaction was blunted in response to infection.

\section{Conclusion}

In conclusion, we identified a secondary inflammatory reaction after US-HIPEC associated to bacterial components in the systemic circulation most likely due to intestinal bacterial translocation. In addition, CRP values were not discriminative after US-HIPEC and patients had a blunted leukocyte reaction in presence of complications.

103

PATIENT-DERIVED ORGANOIDS FROM COLORECTAL PERITONEAL METASTASES AS A PLATFORM FOR INDIVIDUALIZED HYPERTHERMIC INTRAPERITONEAL CHEMOTHERAPY

\author{
O. Kranenburg, I. Ubink, W.M. Van Grevenstein, S.G. Elias, I.H.M. Borel Rinkes
}

University Medical Center Utrecht - Utrecht (Netherlands) 


\section{Objectives}

Cytoreductive surgery combined with hyperthermic intraperitoneal chemotherapy (HIPEC) improves prognosis for patients with peritoneal metastases from colorectal cancer (CRC), but recurrence is common. Mitomycin C (MMC) and oxaliplatin are both commonly used in HIPEC for CRC, but there is no consensus on the optimal treatment regimen. Predictive tools that select patients with peritoneal metastases for treatment with either drug are lacking. We have developed a preclinical model that mimics HIPEC in vitro, and used it to study and compare existing treatment protocols on an individual patient basis, and to explore novel treatment strategies.

\section{Methods}

Organoid technology was used to establish three-dimensional tumour cell cultures from peritoneal metastases obtained through cytoreductive surgery or ascites collection. We seeded small organoids on a bottom layer of extracellular matrix and treated them with increasing doses of hyperthermic $\left(42^{\circ} \mathrm{C}\right)$ mitomycin-C (MMC) or oxaliplatin for 90 and 30 minutes, respectively. Cell viability was measured with CellTiterGlo to determine the half-maximal inhibitory concentration (IC50). Drug-induced cell cycle changes were assessed by flow cytometry, and the effects of combining HIPEC with cell cycle checkpoint inhibitors was evaluated.

\section{Results}

The in vitro HIPEC model was highly reproducibly, resulting in consistent IC50 values over time. We found differences in IC50 values between organoids, but in general 90 minutes of MMC was more effective than 30 minutes of oxaliplatin at clinically relevant drug concentrations. We found that MMC leads to cell cycle arrest in the S-phase. By combining MMC with an inhibitor of ATR, an important player in the DNA damage response that induces S-phase arrest, we could significantly lower the IC50 of MMC in all tested organoids.

\section{Conclusion}

The established in vitro model can be used to study the efficacy of different HIPEC protocols. We propose that peritoneal metastasis-derived organoids could be used to determine the optimal treatment strategy for individual patients. Moreover, it can be used as a platform for development of new, more effective treatment combinations. We suggest that MMC combined with ATR inhibition is a promising novel strategy. By lowering the required dose of MMC, ATR inhibitors could reduce the extensive toxicity related to HIPEC.

104

AN IN VITRO MODEL OF HIPEC: FACILITATING PHARMACOLOGICAL ASSESSMENT IN COLORECTAL PERITONEAL METASTASES

\section{N. Un Nahar, U. Arshad, C. Goldring, P. Sutton}

Centre for Drug Safety Science, Institute of Translational Medicine, University of Liverpool Liverpool (United Kingdom)

\section{Objectives}

Hyperthermic intraperitoneal chemotherapy (HIPEC) is a promising adjunct to cytoreductive surgery in the management of colorectal peritoneal metastases. A deeper understanding of the intrinsic effects of HIPEC, along with its mechanisms of action, efficacy and resistance are still needed. We aimed to establish an in vitro model of HIPEC to facilitate pharmacological assessment in the laboratory setting. 


\title{
Methods
}

Experiments were performed in three human colon cancer cell lines, two of which were isolated from a primary Dukes' C adenocarcinoma (HCT116, DLD-1), and one from a supraclavicular lymph node metastasis (LoVo). A murine colorectal cell line, CT26, was also studied. In addition to their growth in monolayers, HCT116 and CT26 cells were grown in a three dimensional setting as spheroids. The anti-tumour efficacy of oxaliplatin and mitomycin $\mathrm{C}$ as single agents or in combination was assessed, following incubation for 90 minutes at either $37^{\circ} \mathrm{C}$ or $43^{\circ} \mathrm{C}$. Cell viability was analysed using the CellTiter-Glo® chemiluminescence assay (Promega).

\section{Results}

LoVo cells were the most sensitive to the effects of hyperthermia. Administered as a single agent, oxaliplatin (dose range $1-20 \mu \mathrm{M})$ showed enhanced cytotoxicity in LoVo cells at $43^{\circ} \mathrm{C}$ compared to $37^{\circ} \mathrm{C}(p<0.05)$. Interestingly, HCT116 and DLD-1 cells treated with oxaliplatin displayed increased cell viability under hyperthermic conditions when compared with those treated at $37^{\circ} \mathrm{C}$. No difference was seen in CT26 cells. The results observed for HCT116 and CT26 cultured in a monolayer were consistent with those seen in their respective $3 \mathrm{D}$ models. The addition of mitomycin $\mathrm{C}$ to hyperthermia and oxaliplatin showed no synergistic tumoricidal activity in any of the cell lines.

\section{Conclusion}

The combination of hyperthermia and oxaliplatin resulted in an increased tumoricidal effect in the LoVo cell line monolayer. This in vitro model presents an opportunity to further investigate the mechanism, efficacy and the biological basis of HIPEC.

105

$\begin{array}{lllll}\text { CYTOREDUCTIVE } & \text { SURGERY AND } & \text { LAPAROSCOPY-ENHANCED } & \text { HYPERTHERMIC } \\ \text { INTRAPERITONEAL } & \text { CHEMOTHERAPY FOR } & \text { PERITONEAL } & \text { CARCINOMATOSIS: } & \text { FOUR-YEARS } \\ \text { EXPERIENCE } & & & & \end{array}$

\author{
M. Lotti ${ }^{1}$, G. Panyor ${ }^{2}$, M. Marini ${ }^{2}$, E. Vaterlini ${ }^{2}$, M. Giulii Capponi ${ }^{2}$, J. Silvas ${ }^{2}$, C. Bertani ${ }^{2}$, L. Campanati ${ }^{2}$ \\ ${ }^{1}$ Advanced Surgical Oncology Unit - Papa Giovanni XXIII Hospital - Bergamo (Italy), ${ }^{2}$ General Surgery \\ 1 - Papa Giovanni XXIII Hospital - Bergamo (Italy)
}

\section{Objectives}

Cytoreductive Surgery (CRS) and Hyperthermic Intraperitoneal Chemotherapy (HIPEC) is now well recognized as a part of the treatment of peritoneal carcinomatosis in selected patients. While the CRS technique is well accepted, the ideal technique for the delivery of HIPEC is still under debate, the open Coliseum and the closed techniques being the most widespread ones. In 2016 we published a novel procedure, the Laparoscopy-Enhanced Hyperthermic HIPEC technique (LE-HIPEC), that combines the advantages of the open and closed techniques by means of laparoscopy. The perioperative results of our case series are herein presented. 


\title{
Methods
}

Selection criteria for LE-HIPEC were the same used for the open-abdomen HIPEC. Patients and tumor characteristics, intraoperative perfusion and surgery data and perioperative complications were prospectively recorded and retrospectively analyzed.

\section{Results}

Since 2014, 28 consecutive patients (13 male and 15 female) were treated with CRS and LE-HIPEC. Median age was 53 yrs (range $27-73) .16$ patients (57\%) had an ASA score of II, 12 patients $(43 \%)$ had an ASA score of III. Patients were diagnosed with: 10 (36\%) gastric cancer, $7(25 \%)$ colorectal cancer, $6(21.5 \%)$ ovarian cancer, 2 (7\%) pseudomyxoma peritonei, 2 (7\%) adenocarcinoma of the appendix, 1 (3.5\%) mesothelioma. Median PCl score was 12 (range 2-39). 18 patients (64\%) had three or more peritonectomies. 11 patients $(39 \%)$ had three or more visceral resections. 26 patients $(93 \%)$ were treated with cisplatin. During the LE-HIPEC perfusion phase, an average inflow temperature of $42^{\circ} \mathrm{C}$ was required to maintain a $41^{\circ} \mathrm{C}$ temperature within the abdominal cavity. Major perioperative complications were 1 pancreatic fistula that resolved with percutaneous drainage, and 5 acute renal failures ( 1 of them due to blood clots obstructing the ureteral stents), 2 of which resolved spontaneously, 2 resolved after a short course of hemodialysis and 1 progressed to chronic renal failure. No postoperative enteric fistulas and no perioperative mortality were observed.

\section{Conclusion}

In our case series we observed an acceptable rate of perioperative complications. Interestingly, no postoperative enteric fistulas were observed. The LE-HIPEC is a closed procedure that reduces thermal dispersion, providing an homogeneous distribution of heat. A lower inflow temperature can be related with reduced risk of thermal injury to the bowel. To our knowledge, this is the first and largest case series of patients treated with this novel technique.

106

CREATION OF A MODEL OF PERITONEAL CARCINOMATOSIS WITH PANCREATIC HUMAN CELLS FOR TREATMENT WITH HIPEC

\author{
E.P. García Santos, D. Padilla Valverde, P. Villarejo Campos, S. Sánchez García, J. Martín Fernández \\ Hospital General Universitario de Ciudad Real - Ciudad Real (Spain)
}

\section{Objectives}

Creation of an experimental model with athymic rats of pancreatic peritoneal carcinomatosis to know the behavior of chemohyperthermia with Gemcitabine, with respect to a Control group I, of immunocompetent rats, and Control group II, of immunocompetent rats treated with cyclosporine.

\section{Methods}

Tumor pancreatic cell line: The BxPC-3 human line. 
*Model I (3 immunocompetent rats Sprague Dawley®TM, Harlan Laboratories Models, SL, male, 10 weeks, and 250-274gr). We performed peritoneal implantation of $2 \times 106$ human tumor cells in homogenous volumes, with distribution in the 13 abdominopelvic quadrants according to the Peritoneal Carcinomatosis Index $(\mathrm{PCl})$. After five weeks the rats were sacrificed. We established macroscopic quantification of neoplastic volume and histological correlation after complete viscero-peritoneal resection.

*Model II (3 immunocompetent rats experimental animal Sprague Dawley®TM, Harlan Laboratories Models, SL, male, 10 weeks, and 250-274 gr). We performed peritoneal implantation of $20 \times 106$ human tumor cells with homogeneous distribution in volumes, in the 13 abdominopelvic quadrants according to $\mathrm{PCl}$. 48 hours before the inoculation of tumor cells, intraperitoneal administration of cyclosporin (Sandimum $50 \mathrm{mg} / \mathrm{ml}$, Novartis Farmaceutica SA.), $35 \mathrm{mg} / \mathrm{kg} / 24 \mathrm{hrs}$, $(9 \mathrm{mg} / \mathrm{rat}, 0.2 \mathrm{ml}$ ), was carried out daily until completing 50 days. On day 51, the rats were sacrificed. We established macroscopic quantification of neoplastic volume and histological correlation after complete viscero-peritoneal resection.

*Model III (3 immunosuppressed rats, athymic nude rat rnu / rnu, male (Harlan Laboratories) of about 5 weeks with weight 150-200 gr). Implantation of $13 \times 106 \mathrm{cells} / \mathrm{ml}$ was performed, with homogeneous distribution in volumes, in the 13 abdominopelvic quadrants according to ICP. After five weeks the rats were sacrificed. We established macroscopic quantification of neoplastic volume and histological correlation after complete viscero-peritoneal resection.

\section{Results}

In the control models, I and II, macroscopically and histologically, there was no peritoneal implant.

In model III, there was peritoneal carcinomatosis, $\mathrm{PCl}, 14 \pm 3$, with anatomopathological confirmation after radical resection.

\section{Conclusion}

Based on the results obtained, it is a safe and reproducible model of pancreatic peritoneal carcinomatosis for HIPEC.

107

GENETIC LINEAGE TRACING OF PERITONEAL MESOTHELIAL CELLS IN A MOUSE MODEL OF PERITONEAL INJURY

\section{B. Wilm, P. Sutton, T. Wilm}

University of Liverpool, Institute of Translational Medicine - Liverpool (United Kingdom)

\section{Objectives}

The homeostasis of mesothelial layers and their response to adverse stimuli are not very well understood. Specifically, we have only a rudimentary understanding of the molecular mechanisms by which mesothelial cells respond to injury, both in the different anatomical locations that mesothelial layers inhabit, and also regarding individual patient responses.

In order to address some of these questions, we have developed a mouse model that allows detailed tracking of mesothelial cells using an inducer-driven genetic lineage tracing system. 


\title{
Methods
}

We have employed a genetic lineage tracing using the Wilms' tumour gene (Wt1), a specific marker of the developing and adult mesothelial cells, which allows specific fate assessment of mesothelial cells in vivo. Analysis involves whole mount staining and immunofluorescence of tissue sections, as well as image processing and statistical analysis.

\section{Results}

Using the genetic lineage tracing system of mesothelial cells we can demonstrate that after surgical induction of peritoneal injury, Wt1-expressing mesothelial-derived cells change into mesenchymal cells that migrate towards the site of injury where they integrate into the tissues. We are currently analysing the molecular signature of the changes that the mesothelial cells undergo, in order to develop therapeutic interventions.

\section{Conclusion}

Our results demonstrate the robustness of our genetic system, which can be applied to other peritoneal pathologies, including loss of function studies to probe the involvement of specific signalling pathways and the extracellular components of the mesothelial response to adverse stimuli.

108

SYNERGISTIC APOPTOSIS FOLLOWING ENDOPLASMIC RETICULUM STRESS AGGRAVATION IN MUCINOUS COLON CANCER

\author{
H. Choudry, B. Honick, A. Dilly, Y. Lee, D. Bartlett \\ University of Pittsburgh - Pittsburgh (United States)
}

\section{Objectives}

Rapidly proliferating cancer cells rely on endoplasmic reticulum (ER) stress induced unfolded protein response (UPR) pathways to accommodate high protein turnover. Severe and persistent UPR can overwhelm cytoprotective mechanisms of UPR and trigger apoptosis. We hypothesized that mucinous cancers would be especially vulnerable to UPR-mediated apoptosis due to high mucin 2 (MUC2) protein production.

\section{Methods}

In vitro studies were conducted using explant tissue from mucinous (MCC) and non-mucinous (NMCC) colon cancers, normal colon (NC), LS174T cells (MUC2-secreting human colon cancer cells), and dnTCF-LS174T (LS174T cells expressing dominant negative TCF4; differentiated goblet-like cells controlled by Tet-on system). Intraperitoneal tumor model was used for in vivo studies. ER stress aggravators included ER calcium pump inhibitor celecoxib and fatty acid synthase inhibitor orlistat.

\section{Results}

Higher levels of MUC2 and ER stress response proteins, including GRP78/BiP (immunoglobulin heavy chain-binding protein), ATF4 (activating transcription factor 4), and CHOP (C/EBP homologous protein) were detected in MCC tissue and differentiated dnTCF-LS174T cells as compared to NMCC/NC tissue and LS174T cells (qPCR and immunofluorescence). These data suggest a correlation between MUC2 and UPR 
signaling. Celecoxib or orlistat alone decreased cell viability (MTS assay and phase-contrast microscopy), induced apoptosis (flow cytometry) and increased UPR (western blot assay) in a dose-dependent fashion

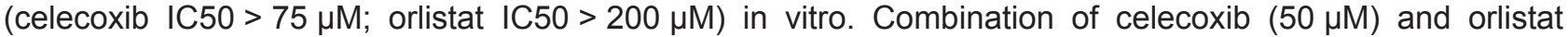
$(100 \mu \mathrm{M})$ demonstrated synergistic loss of cell viability ( $>50 \%$ cell death by MTS assay), induction of apoptosis (flow cytometry) and UPR signaling (western blot assay). Downstream mechanisms involved synergistic induction of NOXA (neutrophil NADPH oxidase factor), cleaved caspases 9 and 3 , and cleaved PARP (poly ADP-ribose polymerase). Chronic intraperitoneal administration of celecoxib + orlistat reduced mucinous tumor growth and improved survival in an intraperitoneal tumor model of MCC.

\title{
Conclusion
}

MCC are vulnerable to treatment with ER stress aggravating drugs due to elevated basal ER stress levels associated with high MUC2 protein turnover. Ongoing studies will determine whether ER stress aggravation by this combination therapy triggers a switch from autophagy to apoptosis.

109

THE TUMOR SUPPRESSOR GENE CDX2 INCREASES 5-FLUOROURACILE RESISTANCE OF COLON CANCER CELLS THROUGH THE ABCC11 TRANSPORTER

\author{
J.B. Delhorme', M.P. Chenard ${ }^{2}$, E. Martin ${ }^{3}$, J.N. Freund ${ }^{3}$, S. Rohr ${ }^{1}$, C. Brigand ${ }^{1}$, I. Gross ${ }^{3}$ \\ ${ }^{1}$ Department of General and Digestive Surgery, Hautepierre Hospital, Strasbourg University Hospital - \\ Strasbourg (France), ${ }^{2}$ Department of Pathology, Hautepierre Hospital, Strasbourg University \\ Hospital - Strasbourg (France), ${ }^{3}$ UMR_S 1113 INSERM / Strasbourg University - Strasbourg (France)
}

\section{Objectives}

Treatment of advanced stages of colorectal cancer (CRC) involves surgery and 5-Fluorouracile (5-FU)-based chemotherapy regimens. Chemoresistance to 5-FU represents a major drawback in CRC management and may be achieved through multiple mechanisms, including active efflux of the drug through overexpression of $A B C$ transporters. The transcription factor CDX2 is a master regulator of intestinal identity that acts as a tumor suppressor in the colon. CDX2 seems to be a prognostic biomarker in stage II and III CRC. CDX2 expression is heterogeneously reduced in CRC and may be important for drug resistance as MDR1/ABCB1 was recently identified as one of its target genes.

\section{Methods}

\section{Results}

By analyzing a meta cohort of 1237 patients treated for CRC and collected from Gene Expression Omnibus at NCBI, we observed a loss of the favorable survival effects of CDX2 expression on DFS for stage III and IV CRC patients treated with systemic chemotherapy. In vitro, we observed that CDX2 expression and intestinal differentiation enhance resistance of colon cancer cell lines towards 5-FU. Using RT-qPCR, we found that chemoresistance of these cells correlates with increased expression of several ABC transporters, namely $A B C B 1$ and $A B C C 11$. Gain and loss of function experiments in colon cancer cells enabled us to show that CDX2 is necessary for the expression of ABCC11, an efflux molecule whose main substrate is 5-FU. Reporter gene and chromatin immunoprecipitation assays confirmed that CDX2 directly regulates the expression of ABCC11 and interacts with its promoter. RT-qPCR analyzes of 66 human healthy samples in 
the digestive tract showed an ABCC11 expression compatible with a CDX2 regulation. Using RT-qPCR, we also observed a correlation between CDX2 and ABCC11 expression levels in a local series of 38 CRC and in the meta cohort of 1237 patients affected with CRC. Finally, we showed that pharmacological inhibition of ABCC 11 by MK-571 improved 5-FU-sensitivity of CDX2-expressing colon cancer.

\title{
Conclusion
}

These data indicate that $A B C C 11$ is a new CDX2 target gene that may contribute to 5-FU chemoresistance in CRC. Further analysis of CDX2 / ABCC11 expression levels and comparison with patients' chemotherapy response in larger cohorts will determine if $A B C C 11$ represents a new prognostic factor or a therapeutic target in CRC.

110

\section{HIPEC-INDUCED ALTERATIONS OF PERITONEAL CELLULAR IMMUNITY}

\author{
J. Franko, M. Andres \\ Mercy Medical Center - Des Moines (United States)

\section{Objectives}

Cytoreductive surgery and hyperthermic intraperitoneal chemotherapy (CS-HIPEC) may alter peritoneal immunologic function and modify tumor-host interaction. We evaluated the feasibility and established elemental characteristics of peritoneal cellular immunology in this pilot project among CS-HIPEC patients.

\section{Methods}

Flow cytometric analysis of peritoneal fluid leukocytes was performed on a convenience sample of eleven patients undergoing CS-HIPEC. Peritoneal fluid (ascites or normal saline washout) $5 \mathrm{cc}$ samples were collected at laparotomy, and then at 30, 60, and 90 minutes of mitomycin $\mathrm{C}$ closed technique perfusion. Following red blood cell lysis a cytometric count analysis using a CD45 ${ }^{+}$gating strategy for CD34 (stem cells), CD15 (granulocytes), CD14 (monocytes), CD3 (T-lymphocytes, CD4 \& CD8 subpopulations), CD3 $\mathrm{CD}^{+}$(NK cells), and CD20 (B-lymphocytes) was performed. The analysis was performed on a Navios Beckman Coulter ten color flow cytometry instrument.

\section{Results}

Total number of leukocytes was $32,034 \pm 29,258 ; 58,254 \pm 36,748,50,825 \pm 34,589$, and $55,409 \pm 34,222$ at laparotomy, and at 30,60 , and 90 minutes of HIPEC, respectively. Trend towards statistically significantly lower cell count was noted among studied time intervals for lymphocytes $(21.3 \pm 26.1,5.8 \pm 8.5,5.6 \pm 7.8$, $4.4 \pm 6.8 \% ; p=0.066)$ and stem cells $(25.5 \pm 45.9,1.0 \pm 0.7,1.3 \pm 1.7,1.0 \pm 0.4 \% ; p=0.073)$. No significance or trends were observed for peritoneal counts of monocytes (baseline count at laparotomy $8.1 \pm 6.9 \%$ of total leucocytes), granulocytes (65.6 $\pm 32.1 \%$ of total leucocytes), NK cells $(5.1 \pm 4.4 \%$ of total lymphocytes), B-lymphocytes $(6.1 \pm 3.6 \%$ of total lymphocytes), CD4 T-cells $(50.3 \pm 11.8 \%$ of total lymphocytes), and CD8 T-cells (26.0 $\pm 10.5 \%$ of total lymphocytes). Similarly, no trends of significance were observed for CD4/CD8 T-lymphocytes ratio (baseline at laparotomy $2.25 \pm 1.08$ ) and neutrophil-tolymphocyte ratio (baseline at laparotomy $43.4 \pm 66.3$ ). Substantial variance of measured peritoneal immunocompetent cells was noted among patients. 


\title{
Conclusion
}

Flow cytometry of peritoneal fluid and HIPEC perfusate is feasible and demonstrates major variance among patients. A follow-up study is ongoing to assess clinical relevance of peritoneal immunologic profile.

I11

EPITHELIAL-MESENCHYMAL TRANSITION MARKERS IN PATIENTS WITH PERITONEAL SURFACE MALIGNANCIES SELECTED FOR CYTOREDUCTIVE SURGERY (CRS) AND HYPERTHERMIC INTRAPERITONEAL CHEMOTHERAPY (HIPEC)
A. Sommariva ${ }^{1}$, M.L. Calabrò ${ }^{2}$
B. Montini' ${ }^{2}$ P. Del Bianco ${ }^{3}$, V. Mozzo ${ }^{2}$
S. Fasolato ${ }^{4}$, M.A. Piano ${ }^{2}$,
C.R. Rossi ${ }^{5}$,

\begin{abstract}
${ }^{1}$ Unit of Surgical Oncology of the Esophagus and Digestive Tract, Veneto Institute of Oncology, IOV IRCCS - Castelfranco Veneto, Tv (Italy), ${ }^{2}$ Immunology and Molecular Oncology, Veneto Institute of Oncology, IOV - IRCCS - Padova (Italy), ${ }^{3}$ Clinical Trials and Biostatistic Unit, Veneto Institute of Oncology, IOV - IRCCS - Padova (Italy), ${ }^{4}$ Unit of Internal Medicine 5 and Hepatology, University of Padova - Padova (Italy), ${ }^{5}$ Surgical Oncology Unit, Veneto Institute of Oncology, IOV - IRCCS - Padova (Italy)
\end{abstract}

\section{Objectives}

Novel biomarkers with predictive/prognostic value are much needed in patients with peritoneal surface malignancies (PSM) considered for CRS+HIPEC. To date, the role and prognostic value of epithelialmesenchymal transition (EMT) parameters in PSM have not been fully investigated. The aim of this study is to analyze the expression profile of EMT-related markers in different PSM histotypes selected for CRS+HIPEC.

\section{Methods}

Tumor samples were obtained during surgery from patients who underwent CRS+HIPEC. Total RNA was extracted from tumor tissues and expression of structural proteins of the epithelial state, specifically ECadherin, ZO-1, and Pancytokeratins (PKRT), as well as transcriptional factors (Snail, Slug, Zeb1, Sip1) and structural and matricellular proteins (N-Cadherin, Vimentin, $\alpha$-SMA, POSTN) of the mesenchymal state were measured by qRT-PCR. The distributions of biomarkers by histology were compared with the Kruskal-Wallis test.

\section{Results}

From 2016 to 2017, 24 patients were enrolled; they were affected by peritoneal mesothelioma, PM (17\%), pseudomyxoma peritonei, PMP (41\%), and other PSM arising from colorectal cancer, CRC (25\%) and epithelial ovarian cancer, EOC (17\%). Among epithelial markers, ZO-1 was more expressed among PM $(p=0.0016)$, that also expressed high levels of PKRT and E-Cadherin. N-Cadherin and Sip1 were significantly more expressed in PM and PMP ( $p=0.0072$ for Sip1; $p=0.0335$ for N-Cadherin), whereas POSTN was prevalently found in CRC and PMP $(p=0.0315)$. CRC were mainly characterized by high ECadherin expression $(p=0.0227)$, whereas higher expression of Sip1 $(p=0.0017), N$-Cadherin $(p=0.0195)$, and ZO-1 ( $p=0.0045)$ was found in PMP. 


\section{Conclusion}

These preliminary results indicate that the analyzed histotypes show different transitional EMT states. PM mainly expressed epithelial markers, confirming their epithelioid histological diagnosis. According to their different clinical behavior, CRC and PMP showed a peculiar EMT profile with a differential expression in E-Cadherin, Sip1, N-Cadherin and ZO-1. The EMT profile of PSM selected for CRS+HIPEC and its prognostic value need to be further evaluated in a larger cohort of patients with a longer follow-up.

112

NON-CANONICAL ACTIVATION OF THE JAK-STAT PATHWAY IS POTENTIALLY DRIVEN BY ACTIVATED INFLAMMATORY-RELATED RECEPTORS IN COLORECTAL PERITONEAL CARCINOMATOSIS

X. Qiu, J. Hendrikson, W.H. Ng, J.W.S. Tan, N.B. Shannon, C.S. Chia, G.H.C. Tan, K.C. Soo, O.L. Kon, C.A.J. Ong, M.C.C. Teo

National Cancer Centre Singapore - Singapore (Singapore)

\section{Objectives}

The accumulation of ascitic fluid is one of the key complications in peritoneal carcinomatosis (PC) and often an indication of poor prognosis in PC patients. Increasingly, reports have shown that ascites constitute an important tumour milieu that accentuates tumorigenesis and metastasis. However, the molecular basis of the role of ascites in cancer development remains unclear. In this study, we aim to achieve the following:

1) Identify the ascites-associated signaling pathways that promote tumour biological behavior 2) Decipher putative upstream activators of these pathways via cytokine arrays and phosphor-Receptor Tyrosine Kinase (RTK) Array.

\section{Methods}

Ascites was collected during cytoreductive surgery from 3 PC patients and was processed to obtain sterile cell-free ascites for subsequent experiments. 2 metastatic colorectal cell lines were treated using the malignant ascites $(n=3)$ derived from colorectal origin. RNA was extracted from the ascites treated cells and subjected to microarray analysis. Gene set enrichment analysis (GSEA) was applied to the gene expression data. Matched ascites was subjected to cytokine and phospho-Receptor Tyrosine Kinase (RTK) Array.

\section{Results}

GSEA of the gene expression data derived from the ascites treated cells identified several pathways that were significantly enriched across all the ascites treated cells. While cell-line specific pathways were identified in the analysis, consistent across both cell lines was the upregulation of key pathways such as JAK-STAT signaling and IFN- $\gamma$ response (Normalized enrichment score $1.31 \quad(p=0.079)$ and 1.36 $(p=0.021)$ respectively). Highlighting the ascites-induced inflammatory phenotype, analysis of the RTK array demonstrated activated inflammatory-related receptors like Zap70 and EPHA7. Interestingly, JAK receptors were found to be inactive. Moreover, further interrogation of the secretome in the ascites using cytokine array revealed that the common activating ligand IL6 was not enriched in our data, hence, suggesting that the augmented STAT signaling could possibly be activated via an alternative mechanism. 


\title{
Conclusion
}

We identified a group of patients who may potentially benefit from personalized therapy targeting the noncanonical pathway of the JAK-STAT signaling.

113

\section{NOVEL ORGANOID MODELS TO EVALUATE EFFICACY OF IMMUNOTHERAPY FOR COLORECTAL PERITONEAL METASTASES}

\author{
V. Narasimhan, T. Pham, M. Michael, A. Heriot, R. Ramsay \\ Peter MacCallum Cancer Centre - Melbourne (Australia)

\section{Objectives}

Colorectal cancer (CRC) is the third most common cancer worldwide. Up to half the patients with CRC will develop metastatic disease, with a 5 -year survival of $13.5 \%$. The peritoneum is a common site for metastases, but carries the worst prognosis among all sites of metastases. Most patients with colorectal peritoneal metastases (CRPM) are not operable, leaving only systemic chemotherapy as a treatment option. With systemic chemotherapy alone, only $4 \%$ of patients with CRPM are alive at 5 years.

Immunotherapy, while effective in a number of cancers, remains unexplored in peritoneal disease. Here, we aim to explore the immune landscape of CRPM, and evaluate the role of immunotherapy in CRPM using a novel organoid based model.

\section{Methods}

Fresh tissue from microsatellite stable (MSS) patients with colorectal peritoneal metastases was used for flow cytometry (FACS) analysis to evaluate the immune cell infiltration. Tissue was further processed to develop robust organoid models. Simultaneous patient matched tumour infiltrative lymphocytes (TILs) were cultured from fresh tissue and expanded with interleukin-2 (IL-2). Once organoids were robust and well established, they were co-cultured in a novel cytotoxic assay with various concentrations of TILs to assess the cytotoxic capacity of the lymphocytes. Organoids were also co-cultured with TILs and the addition of an anti PD-1 antibody to assess the role of checkpoint blockade.

\section{Results}

FACS analysis revealed a heterogenous population of immune cells with a significant $T$ cell infiltrate. There was an even mix of CD4 and cytotoxic CD8 cells. However, there were unexpectedly high levels of Tregulatory cells, comprising up to $15 \%$ of CD4 cells. There were high levels of T cell memory and activation markers such as CD45RO and HLA-DR. PD-1 expression on T cells, whilst variable, was up to $75 \%$ in some patients, suggesting a possible role for anti PD-1 antibody therapy. Co-culture with organoids and TILs in a novel live cell scanning assay demonstrated successful organoid killing by the TILs, with high levels of interferon- $\delta$ detected in the supernatant. In patients with high T cell PD-1 expression on FACS, addition of a PD-1 antibody revealed significantly increased cytotoxic killing of the organoids. 
Supplemental Congress Abstracts - PSOGI 2018, Paris, September 9-11, 2018 • https://doi.org/10.1515/pap-2018-7024

\title{
Conclusion
}

This novel assay provides functional evidence of the potential of immunotherapy as an adjunctive treatment for CRPM. These early results need to be expanded with a larger cohort, and validated in an in-vivo setting.

I14

\section{PRECISION MEDICINE: DEVELOPMENT OF A NOVEL ORGANOID BASED PLATFORM FOR PERSONALISED THERAPY IN COLORECTAL PERITONEAL METASTASES. ES}

\author{
V. Narasimhan, T. Pham, M. Michael, R. Ramsay, A. Heriot \\ Peter MacCallum Cancer Centre - Melbourne (Australia) \\ Objectives
}

Colorectal cancer $(C R C)$ is the third leading cause of cancer-related mortality worldwide. Up to half the patients with CRC develop metastatic disease, with an associated five-year survival of $13.5 \%$. The peritoneum is a common site for metastases, but carries the worst prognosis among all sites of CRC metastases. The majority of patients with colorectal peritoneal metastases (CRPM) are inoperable, leaving chemotherapy as the only treatment option. With best available chemotherapy, these patients can achieve a median survival of 12-16 months. We currently treat patients with generic chemotherapy drugs without any knowledge of the patient's tumour sensitivity to the prescribed drug. It is plausible that many treatment failures in patients with CRPM are due to this generic model of treatment.

We aim to develop a novel platform incorporating robust pre-clinical models, genomic and transcriptomic data with throughput drug sensitivities for personalised therapy in patients with unresectable CRPM.

\section{Methods}

Laparoscopy was performed for all patients to assess the peritoneal carcinoma index (PCI). Tumour biopsies were taken at laparoscopy to grow organoids. Organoids were validated with Short tandem repeat (STR) analysis and immunohistochemistry. Organoids were expanded for DNA and RNA extraction for whole exome sequencing (WES) and RNASeq. Organoids were subsequently put through a throughput drug sensitivity platform involving over 50 TGA (Therapeutic Guidelines Australia) approved drugs.

\section{Results}

Organoids were successfully grown and validated in $80 \%$ of cases from laparoscopic biopsies. Genomic and transcriptomic data helped define the genomic landscape of CRPM. It helped identify targetable mutations and pathways alterations. Organoid throughput drug sensitivity analysis revealed that many CRPM organoids are resistant to commonly used chemotherapies such as 5-FU, Oxaliplatin and Irenotecan. For most organoid lines, the top three sensitive drugs did not include the drug the patient was currently on. All these results were obtained and analysed within a six-week period.

\section{Conclusion}

We have successfully developed a novel organoid based platform combining genomic, transcriptomic and drug sensitivity data to personalise chemotherapy for unresectable CRPM. The next step to advance this promising pre-clinical data is a clinical trial to confirm the feasibility and efficacy of this novel platform to help improve patient outcomes. 
I15

\title{
VARIATION OF CONCENTRATION OF OXALIPLATIN IN BSA BASED ADMINISTRATION OF OXALIPLATIN DURING HIPEC
}

\author{
V. Verwaal, J. Funder, L. Iversen, M. Møller Sørensen \\ Aarhus University Hospital - Aarhus (Denmark) \\ Objectives
}

Oxaliplatin based protocols have become the mainstream treatment in HIPEC. There are currently a two way of calculating the dose of chemotherapy given. One protocol aims to $460 \mathrm{mg}$ oxaliplatin in $2 \mathrm{l} / \mathrm{BSA}$. The other aims at $260 \mathrm{mg}$ oxaliplatin per BSA. Recently a standard protocol for HIPEC with oxaliplatin was introduced. This protocol was based on doses of oxaliplatin on BSA. In this study we looked at the variation in concentration of oxaliplatin at the beginning of the HIPEC.

\section{Methods}

Between October 2016 and January 2018, a total of 64 patients underwent a HIPEC with oxaliplatin. Inclusion was peritoneal metastasis of colon or rectal cancer. Exclusion was toxicity of previous given oxaliplatin. The doses of oxaliplatin was calculated on the BSA, $260 \mathrm{mg} / \mathrm{m}^{2}$ was given with a maximum of $520 \mathrm{mg}$ if the BSA $>2.0 \mathrm{~m}^{2}$. The volume of the perfusate was measured by perfusion machine and based on the weight of it. BSA was measured using the Du Boise Formula. The amount of oxaliplatin given was measured by the pharmacy department based on the BSA.

\section{Results}

The 64 patients had a median weight of $79 \mathrm{~kg}$ (range 50 to $123 \mathrm{~kg}$ ) and a median height of $175.5 \mathrm{~cm}$ (range 151 to $191 \mathrm{~cm}$ ). The median BSA was $1.92 \mathrm{~m}^{2}$ (range 1.48 to $2.43 \mathrm{~m}^{2}$ ). The median dose of oxaliplatin was $499.85 \mathrm{mg}$ (range 384.0-529.8 mg). The total volume of the perfusate was median $7003 \mathrm{ml}$ (range 3005$7016 \mathrm{ml}$ ), the concentration of oxaliplatin was median $74 \mathrm{mg} / \mathrm{l}$ perfusate with a range from 38 to $173 \mathrm{mg} / \mathrm{l}$. There were 14 protocol violations. These violations were all overdosing if the BSA was over 2.0.

From this study the concentration has a large range from 38 to $173 \mathrm{mg} / \mathrm{l}$. This range is mainly due to the wide range in the used perfusate. If one would have based the calculation on a constant concentration the range of the total amount of oxaliplatin would have been $(230 \times 3.005 \mathrm{I}=) 691$ to $(230 \times 7016=) 1613 \mathrm{mg}$. The normal systemic dose of oxaliplatin is $100 \mathrm{mg} / \mathrm{m}^{2}$. In our population this would had a range from 148 to $243 \mathrm{mg}$.

The question if the concentration or the total amount of chemotherapy drive the cytotoxic effect has not been solved yet. However, in the systemic chemotherapy treatment is the amount based on BSA.

\section{Conclusion}

The concentration range of oxaliplatin in the perfusate has a wide range if one bases the amount of chemotherapy on the BSA. If one bases the calculation on concentration the total amount of chemotherapy varies widely. 
116

\title{
MOLECULAR AND GENETIC MARKERS IN APPENDICEAL MUCINOUS NEOPLASMS - A SYSTEMATIC REVIEW
}

\author{
H. Mogal ${ }^{1}$, A. Stein ${ }^{1}$, T.C. Gamblin ${ }^{1}$, C. Clarke ${ }^{1}$, S. Tsai ${ }^{1}$, J. Thomas ${ }^{2}$, B. George ${ }^{2}$ \\ ${ }^{1}$ Department of Surgery, Division of Surgical Oncology, Medical College of Wisconsin - Milwaukee \\ (United States), ${ }^{2}$ Department of Medicine, Division of Hematology/Oncology, Medical College of \\ Wisconsin - Milwaukee (United States)
}

\section{Objectives}

Significant advances have been made in the histologic classification of Appendiceal Mucinous Neoplasms (AMNs), however, the role of somatic alterations (SAs) is evolving. Using a systematic review of the literature, we sought to identify putative SAs, representative of the histologic entities that comprise AMNs and SAs that are associated with aggressive clinical phenotypes.

\section{Methods}

MEDLINE/PubMed was searched for studies on AMNs including molecular markers or genomic alterations, published between 1990 and 2017. Based on available consensus guidelines, studies were grouped under low-grade and high-grade histological type for primary and metastatic tumors.

\section{Results}

A total of 22 studies involving 970 tumor samples (primary and metastatic) were identified. PCR, Sanger and Next Generation Sequencing were the commonly used methods for DNA sequencing. Median age ranged between 49-71. Six studies involving 135 primary low-grade AMNs (including well differentiated adenocarcinoma) identified KRAS $(71.1 \%)$ as the predominant SA. Five of these studies noted SAs in GNAS in $44.6 \%$ of 65 LAMNs. KRAS was identified in $74 \%$ of 11 studies with 187 low-grade PMP (pseudomyxoma peritonei). In eight of these studies, GNAS SAs were noted in $53 \%$ of 83 tumors. Five studies noted TP53 SAs in only $5.9 \%$ of 34 tumors. High-grade AMNs (mucinous adenocarcinomas NOS, moderate to poorly differentiated adenocarcinomas, adenocarcinoma with signet ring cells and Goblet cell carcinoids) demonstrated significantly lower SAs in KRAS (45.2\% of 436 tumors in 10 studies) and GNAS (26.4\% of 110 tumors in five studies) and higher SAs in TP53 (26.7\% of 120 tumors in five studies). In high-grade PMP, SAs were noted in KRAS (70.5\% of 122 tumors in eight studies), GNAS (41.3\% of 46 tumors in six studies) and TP53 (44\% of nine tumors in two studies). PIK3CA, ATM, APC, AKT1 and SMAD4 were other less frequent $(<15 \%)$, albeit potentially relevant mutations identified.

\section{Conclusion}

While KRAS and GNAS are the predominantly identified SAs in primary and metastatic low-grade AMNs, they are less frequently noted in high-grade tumors. Conversely, TP53 is rare in low-grade but frequently altered in high-grade primary and metastatic AMNs. Although SAs may provide valuable insights into tumor heterogeneity and variability in tumor biology, larger studies utilizing clinically annotated genomic databases from multi-institutional consortiums may improve their identification and clinically applicability in the management of AMNs. 
I17

CRS AND OXALIPLATIN-BASED HIPEC IN A RAT MODEL OF COLORECTAL PERITONEAL SURFACE MALIGNANCY: ESTABLISHING SENSITIVITY AND MAXIMUM TOLERATED DOSE IN A VALIDATED ASSAY

\section{Lemoine ${ }^{1}$, P. Sugarbaker ${ }^{2}$, K. Van Der Speeten ${ }^{1}$}

${ }^{1}$ Dpt of Medicine and Life Sciences, Hasselt University; Dpt of Surg. Oncol., Ziekenhuis Oost-Limburg - Hasselt, Genk (Belgium), ${ }^{2}$ MedStar Washington Hospital Center - Washington DC (United States)

\section{Objectives}

At present, the combination therapy of CRS and HIPEC is considered standard of care for colorectal peritoneal surface malignancy (PSM) treatment. Although, there is a near universal standardization regarding the CRS, we are still lacking a much-needed standardization amongst the various IP chemotherapy treatment modalities. We should rely on validated analytical assays and well-designed preclinical studies to build pharmacologic data towards improved and standardized HIPEC regimens. Until today, a validated analytical assay to quantify oxaliplatin was not available. The syngeneic CC-531 cell line, injected in the WAG/Rij rat, is a widely used animal model to study colorectal PSM. We report on its use to evaluate maximum tolerated dose (MTD) of oxaliplatin and its sensitivity, using a validated assay.

\section{Methods}

Sensitivity of the CC-531 cell line for oxaliplatin was evaluated by means of the MTT assay. Twenty seven WAG/Rij rats were IP injected with the CC-531 cell line. Peritoneal disease burden was evaluated on 6,7 and 8 days post cell line injection, using the modified PCl score. The MTD of oxaliplatin, in $0.9 \% \mathrm{NaCl}\left(2 \mathrm{~L} / \mathrm{m}^{2}\right)$ as carrier solution, during a 30-minute HIPEC was evaluated for the following doses: $40-60-100-150 \mathrm{mg} / \mathrm{m}^{2}$. The MTD was defined as the highest non-lethal dose of oxaliplatin, at which the humane endpoints were not reached ( $20 \%$ relative weight loss, for 3 consecutive days within 2 weeks).

\section{Results}

Cell viability of the CC-531 cells decreased with increasing oxaliplatin concentration, with a mean cell viability of $55.57 \% \pm 2.35$ at a concentration of $75 \mu \mathrm{g} / \mathrm{mL}$ oxaliplatin. Time span needed to establish a clinical representative image of PSM was 8 days post cell line injection. MTD was $150 \mathrm{mg} / \mathrm{m}^{2}$ oxaliplatin in $2 \mathrm{~L} / \mathrm{m} 20.9 \% \mathrm{NaCl}$ carrier solution.

\section{Conclusion}

The CC-531 cell line was demonstrated to be sensitive for oxaliplatin in a colorectal PSM rat model. MTD was established at $150 \mathrm{mg} / \mathrm{m}^{2}$ oxaliplatin in $2 \mathrm{~L} / \mathrm{m} 20.9 \% \mathrm{NaCl}$ carrier solution. 
118

KRAS AND GNAS - DRUGGABLE TARGETS IN PSEUDOMYXOMA PERITONEI?

\author{
A.T. Kristensen ${ }^{1}$, C. Lund-Andersen ${ }^{1}$, L. Kyllingstad ${ }^{2}$, Y. Andersson ${ }^{1}$, I.S. Frøysnes ${ }^{1}$, \\ T.W. Abrahamsen ${ }^{1}$, P. Krolokowski ${ }^{1}$, I. Bergheim ${ }^{3}$, B. Davidson ${ }^{4}$, S. Dueland ${ }^{5}$, S.G. Larsen ${ }^{6}$, \\ K. Flatmark ${ }^{7}$
}

${ }^{1}$ Department of Tumorbiology, Norwegian Radium Hospital, Oslo University Hospital - Oslo (Norway), ${ }^{2}$ Department of Tumorbiology, Norwegian Radium Hospital, Oslo University Hospital. Institute of Clinical Medicine, University of Oslo - Oslo (Norway), ${ }^{3}$ Department of Cancer Genetics, Norwegian Radium Hospital, Oslo University Hospital - Oslo (Norway), ${ }^{4}$ Department of Pathology, Norwegian Radium Hospital, Oslo University Hospital. Institute of Clinical Medicine University of Oslo - Oslo (Norway), ${ }^{5}$ Department Oncology, Norwegian Radium Hospital, Oslo University Hospital - Oslo (Norway), ${ }^{6}$ Department of Gastroenterological Surgery, Norwegian Radium Hospital, Oslo University Hospital - Oslo (Norway), ${ }^{7}$ Department of Tumorbiology, Department of Gastroenterological Surgery, Oncology, Norwegian Radium Hospital, Oslo University Hospital, Oslo, Norway. Institute of Clinical Medicine, University of Oslo - Oslo (Norway)

\title{
Objectives
}

Pseudomyxoma peritonei (PMP) is a rare cancer originating in mucinous tumors of the appendix and is characterized by tumor spread to the surfaces of the peritoneal cavity. Surgery followed by hyperthermic intraperitoneal chemotherapy (HIPEC) may cure patients, but new therapeutic options are needed for patients who cannot be cured by surgery. An important limitation for improving PMP treatment is the lack of accurate molecular diagnosis, preventing understanding of disease mechanisms and identification of therapeutic targets. A challenge in this respect is that the tumor tissue often has very low tumor cell content.

\section{Methods}

DNA from tumor and blood samples from 80 PMP patients treated at the Norwegian Radium Hospital was analyzed by targeted next-generation sequencing (Ion AmpliSeqTM Cancer Hotspot panel v2) with high sequencing depth. Data was processed by the Torrent Suite Variant Caller, using panel-customized parameters. Every detected mutation was manually reassessed using Integrative Genomics Viewer. Analyses are ongoing and data from 46 cases is presented in this abstract.

\section{Results}

Somatic mutations were detected in 32 samples, even in cases with extremely low tumour content $(<1 \%$; $42 / 80$ samples), but in $30 \%(14 / 46)$ no mutation was found. The most frequently mutated genes were KRAS $(65 \%)$ and GNAS (59\%), followed by SMAD4 (9\%), and occasional mutations in BRAF, PIK3CA, NRAS, $A T M, C T N N B 1, E G F R, K D R$, and SMO. Associations between mutations and clinical outcome will be analyzed and presented.

\section{Conclusion}

We identified mutations in most PMP patient samples using next-generation targeted sequencing, even in cases with extremely low tumor content. The most frequently mutated genes were KRAS and GNAS and these proteins would represent valid therapeutic targets in PMP if inhibitors were available. For cases without detectable mutations, a broader sequencing approach is recommended, but may be hampered by low tumor cell content in samples. 
119

BRAF V600E MUTATION - A PROGNOSTIC BIOMARKER IN PERITONEAL METASTASIS FROM COLORECTAL CANCER

C. Lund-Andersen ${ }^{1}$, A. Kristensen ${ }^{1}$, L. Kyllingstad ${ }^{2}$, I.S. Frøysnes ${ }^{1}$, T.W. Abrahamsen ${ }^{1}$, P. Krolowski ${ }^{1}$, I. Bergheim ${ }^{3}$, B. Davidson ${ }^{4}$, S. Dueland ${ }^{5}$, S.G. Larsen ${ }^{6}$, K. Flatmark ${ }^{7}$

${ }^{1}$ Department of Tumorbiology, Norwegian Radium Hospital, Oslo University Hospital - Oslo (Norway),

${ }^{2}$ Department of Tumorbiology, Norwegian Radium Hospital, Oslo University Hospital. Institute of Clinical Medicine, University of Oslo - Oslo (Norway), ${ }^{3}$ Department of Cancer Genetics, Norwegian Radium Hospital, Oslo University Hospital - Oslo (Norway), ${ }^{4}$ Department of Pathology, Norwegian Radium Hospital, Oslo University Hospital. Institute of Clinical Medicine, University of Oslo - Oslo (Norway), ${ }^{5}$ Department of Oncology, Norwegian Radium Hospital, Oslo University Hospital. Institute of Clinical Medicine, University of Oslo - Oslo (Norway), ${ }^{6}$ Department of Gastroenterological Surgery, Norwegian Radium Hospital, Oslo University Hospital - Oslo (Norway), ${ }^{7}$ Department of Tumorbiology, Department of Gastroenterological Surgery, Norwegian Radium Hospital, Oslo University Hospital. Institute of Clinical Medicine, University of Oslo - Oslo (Norway)

\section{Objectives}

Peritoneal metastasis from colorectal cancer (PM-CRC) is a major cause of CRC-related morbidity and mortality. Surgery followed by hyperthermic intraperitoneal chemotherapy (HIPEC) may prolong survival and even cure some patients. However, the treatment is demanding and selection of patients likely to benefit from the treatment is crucial. Increased knowledge of the molecular composition of PM-CRC is needed to identify biomarkers for treatment selection and new therapeutic targets.

\section{Methods}

DNA from tumor and blood samples from 104 PM-CRC patients treated at the Norwegian Radium Hospital was analyzed by targeted next-generation sequencing (Ion AmpliSeqTM Cancer Hotspot panel v2). Data was processed by the Torrent Suite Variant Caller, using panel-customized parameters. Every detected mutation was manually reassessed using Integrative Genomics Viewer. Associations with clinical patient characteristics and treatment outcome were analyzed.

\section{Results}

Mutations were detected in 97/104 cases. The most frequently mutated genes were TP53 (52\%), KRAS (40\%), BRAF (26\%), APC (25\%), SMAD4 (17\%) and PIK3CA (14\%), which all are commonly mutated in CRC. Interestingly, the APC mutation frequency was unusually low compared to previous reports from primary CRC and colorectal liver metastases ( $76 \%$ and $61 \%$, respectively). In contrast, BRAF mutations were more frequent, and only V600E mutants were detected. The presence of BRAF mutation was associated with inferior disease-free (DFS) and overall survival (OS) compared to non-mutated cases (median DFS 6.0 vs. 14.1 months, $p=0.02$; median OS 22.6 vs. 42.8 months, $p=0.02$, respectively).

\section{Conclusion}

$B R A F$ mutation should be further investigated as a prognostic biomarker in PM-CRC and may also identify patients eligible for participation in trials investigating therapeutic regimens containing $B R A F$ inhibitors. 
120

COLORECTAL PERITONEAL ORGANOIDS: ESTABLISHMENT, VALIDATION AND UTILITY AS A PRE-CLINICAL MODEL OF DISEASE

\author{
V. Narasimhan, T. Pham, A. Heriot, R. Ramsay \\ Peter MacCallum Cancer Centre - Melbourne (Australia) \\ Objectives
}

Colorectal cancer (CRC) is the third leading cause of cancer-related mortality worldwide. Up to half the patients with CRC develop metastatic disease, with an associated five-year survival of $13.5 \%$. The peritoneum is a common site for metastases, but carries the worst prognosis. The majority of patients with colorectal peritoneal metastases (CRPM) are inoperable, leaving chemotherapy as the only treatment option. With chemotherapy alone, only $4 \%$ of patients are alive in 5 years. Clearly, there is an urgent need to investigate newer avenues of treatment for this group of patients with unresectable CRPM.

To evaluate efficacy of any treatment in a pre-clinical setting, one needs robust pre-clinical models of disease. We now know that traditional cell lines do not recapitulate the native tumour very well, and can drift to a different phenotype based on the dominant clone. Murine models, while effective in maintaining the tumour microenvironment, are time exhaustive, taking many months to develop. Organoids are a newer preclinical model that bridge the gap between cell lines and mouse models. Organoids are three dimensional cell cultures that recapitulate the native tumour heterogeneity. They can be rapidly established, are stable over passages, can be safely cryopreserved and are portable across institutes. Organoid models can be used to develop a personalised model of disease in a short time frame.

\title{
Methods
}

We used fresh colorectal peritoneal metastatic tumour tissue to develop organoid models of CRPM. Our protocol was a modification of the published Cleavers protocol.

\section{Results}

Organoids were validated by short tandem repeat (STR) analysis and immunohistochemistry (CK20, CDX2). Tumourigenicity was confirmed by implanting organoids into immunocompromised (NSG) mice. Orthotopic models were developed by implanting organoids into the peritoneum of NSG mice. Organoid derived xenografts were validated in a similar way by immunohistochemistry including use of anti-mitochondrial antibody. Immune profiling of the organoids were performed by challenge with interferon gamma for 48 hours and then staining them with PD-L1 and MHC-I. This served to assess whether altered regulation of certain ligands is an immune evasion mechanism used by the tumour to evade immune cell based killing.

\section{Conclusion}

This stringent method of validation confirms successful development of CRPM organoids, which can be utilised for genomic, cell signalling, immunologic, or cytotoxic studies. 
121

\title{
CHARACTERIZATION AND CONSEQUENCES OF HYPOGAMMAGLOBULINEMIA AFTER CYTOREDUCTIVE SURGERY AND HYPERTHERMIC INTRAPERITONEAL CHEMOTHERAPY
}

\author{
C. Soldevila-Verdeguer, J.J. Segura-Sampedro, N. Esteve-Pérez, A. Craus-Miguel, Á. Molina-Fuentes, \\ A. Repetto, M. Guillot-Morales, J. Milà-Llambí, J. Pons-De Ves, R. Morales-Soriano \\ Hospital Universitario Son Espases - Palma (Spain)
}

\section{Objectives}

The immune system's function can be compromised under circumstances such as a neoplastic process or chemotherapeutic treatments, leading to an increase of infectious complications. The aim of this study was to investigate if peritoneal carcinomatosis (PC) treatment with cytoreductive surgery (CRS) in association with hyperthermic intraperitoneal chemotherapy (HIPEC) causes secondary hypogammaglobulinemia and elucidate its relationship with postoperative complications.

\section{Methods}

A retrospective observational study including patients undergoing CRS-HIPEC was conducted between March 2014 and May 2017. Patients with a history of previous CRS-HIPEC and lost to follow-up patients where excluded. Hipogammaglobulinemia was correlated with postoperative morbidity and length of stay.

\section{Results}

Seventy patients where included, $60 \%$ women (42) and $40 \%$ men (28), with a median age of 59 . Most of the patients were treated for PC following a primary colon carcinoma $(50 \%)$ and primary ovarian carcinoma (36\%). The median peritoneal carcinomatosis index (ICP) was 12. In $96 \%$ of patients there was a lower lgG concentration after surgery with a median decrease of $400 \mathrm{mg} / \mathrm{dL}$. The increase of ICP correlated with the decrease in IgG levels ( $r-0,47, p$ 0.01). Forty-two patients $(52 \%)$ reached hypogammaglobulinemia (IgG $<500 \mathrm{mg} / \mathrm{dL}$ ), $36 \%$ of them presented major surgical morbidity (Clavien-Dindo III-V) whereas $18 \%$ of the patients without hypogammaglobulinemia, although there was no statistical significance. The median length of stay was 24 days in the first group and 14 in the second $(p 0,03)$. Twenty-five patients developed infectious complications, they had lower IgG levels than patients without infectious morbidity ( $p 0.02)$.

\section{Conclusion}

This is the first time to our best knowledge that hypogammaglobulinemia after CRS-HIPEC has been described and related to postoperative morbidity. Our study shows a higher rate of major complications and length of stay in patients developing hypogammaglobulinemia after CCR-HIPEC. The physiopathology of this phenomenon and it's association with surgical outcomes needs further characterization and research. 
122

ASSESSMENT OF SENSITIVITY OF TUMOR TISSUE TO CYTOTOXIC DRUGS AS AN AID IN THE SELECTION OF AN ANTICANCER DRUG USED IN THE HIPEC PROCEDURE.

\author{
T. Jastrzebski ${ }^{1}$, H. Heidecke ${ }^{2}$, T. Polec ${ }^{1}$ \\ ${ }^{1}$ Surgical Oncology Department. Medical University Gdansk - Gdansk (Poland), ${ }^{2}$ Zentrum für \\ Molekulare Onkologie GmbH - Luckenwalde (Germany)
}

\title{
Objectives
}

The aim of this study was to assess the sensitivity of tumour tissue to cytostatics in order to individualize the approach to the treatment of patients with metastases to the peritoneum and the inclusion of targeted chemotherapy based on the results of this study

\section{Methods}

The study included 22 patients diagnosed with abdominal tumours with metastases to the peritoneum. The sensitivity assessment of tumour tissue to cytostatics was performed by means of the in vitro adenosine triphosphate-based chemotherapy response assay (ATP-CRA).

\section{Results}

Out of 11 patients with ovarian cancer the sensitivity to drugs used in HIPEC procedure in this type of tumour (cisplatin) was observed in 4 patients (4/11-64\%). In 3 of cases, sensitivity to doxorubicin was also observed. In turn, sensitivity to paclitaxel was observed in 9/11 patients (82\%), 7 of whom the sensitivity was defined as strong and as poor in 2. In the 7 patients with bowel cancer, appendix cancer and peritoneal pseudomyxoma (PMP) where the drug used in the HIPEC procedure was Mitomicin C or oxaliplatin, no sensitivity to these cytotoxic drugs was observed in the tumour tissue. Instead, sensitivity to paclitaxel was observed in $4 / 7$ patients (57\%), including 3 patients with pseudomyxoma peritonei.

\section{Conclusion}

The test results indicate a lack of sensitivity of tumour tissue of metastases to the peritoneum of colorectal cancer and PMP to cytotoxic drugs generally used in the HIPEC procedure (mitomicin C, oxaliplatin). The results if this study suggest that the drug of choice in the HIPEC procedure should be paclitaxel.

123

OXALIPLATIN TISSULAR PENETRATION DURING HEATED INTRAOPERATIVE CHEMOTHERAPY (HIPEC) MAY IMPACT SURVIVAL

\footnotetext{
O. Sgarbura ${ }^{1}$, J. Bianga ${ }^{2}$, M. Larroque ${ }^{3}$, A. Bouslimani $^{3}$, N. Bec ${ }^{3}$, C. Larroque ${ }^{3}$, F. Quenet $^{3}$

${ }^{1}$ Institut of Cancer Montpellier - Montpellier (France), ${ }^{2}$ LCABIE, UMR 5254, Hélioparc - Pau (France), ${ }^{3}$ Institut de Recherche en Cancérologie - Montpellier (France)
} 


\title{
Objectives
}

Heated Intraoperative Chemotherapy (HIPEC) is used as a complement to complete cytoreductive surgery in several conditions associated with peritoneal carcinosis(PC). Oxaliplatine is used as the main molecule in HIPEC for PC of gastrointestinal origin. The aim of the study is to evaluate whether cell penetration of oxaliplatine measured by matrix-assisted laser desorption ionization (MALDI) and laser ablation ICP mass spectrometry is linked to survival.

\section{Methods}

For eight patients with PC of colorectal and gastric origin, tumoral specimens were collected at the end of HIPEC and sent for preparation as tissues frozen in liquid nitrogen at $-80^{\circ} \mathrm{C}$. $10 \mu \mathrm{m}$-slices were mounted in indium-tin oxide and analyzed wit MALDI and LA-ICP MS. The tissular penetration of the drug was measured as the percentage of the section area presenting Pt-metabolites. Clinical, operative and survival data were collected. Statistical analysis was performed using correlation and non-parametric tests.

\section{Results}

The eight patients had a M:F ratio $=2: 6$ and an average age of $58,25( \pm 12,15)$ years. There was one case of gastric adenocarcinoma and seven of colorectal adenocarcinoma with an average $\mathrm{PCl}$ of 11,88 $\pm 5,59$. Median overall survival was 70,75 months. Whilst correlation between PCl and survival was mild $(r=0,362)$, correlation between Pt-penetrated tissue and overall survival(OS)/progression-free survival(PFS) was strong $(r=0,689 ; r=0,753)$. P-value was non-significant due to sample size.

\section{Conclusion}

This pilot study suggests that there is a link between oxaliplatine tissue penetration during HIPEC for gastrointestinal cancer PC and both OS and PFS but more samples are needed in order to potentially attain statistical significance.

124
A FEASIBILITY STUDY OF OXALIPLATIN + DOXORRUBICIN ADMINISTRATED ON THE BASIS OF CONCENTRATION IN THE PERFUSATE SOLUTION DURING HIPEC FOR THE TREATMENT OF PERITONEAL METASTASES (PM)

\author{
P. Bretcha-Boix, V. Escudero, A. Catalan, M. Sureda, J. Farre-Alegre, M. Duarte, A. Paz, A. Brugarolas \\ Hospital Quironsalud Torrevieja - Torrevieja (Spain)
}

\section{Objectives}

To evaluate the feasibility of adding doxorubicin to oxaliplatin, both dosed with the concentration method (mg/L), in order to keep the homogeneity in concentration during HIPEC

\section{Methods}

After cytoreductive surgery, patients (pts) were treated using a glucose $5 \%$ solution instilled to $42-43^{\circ} \mathrm{C}$ within the peritoneal cavity in a perfusion period of 30 minutes. Oxaliplatin was dosed at a concentration of $200 \mathrm{mg} / \mathrm{L}$ and doxorubicin at $15 \mathrm{mg} / \mathrm{L}$. 
To evaluate the exposure to the drugs, samples of peritoneal fluid and plasma were extracted at different times. Plasma and peritoneal fluid oxaliplatin concentrations were quantified by atomic absorption spectroscopy and doxorubicin by liquid chromatography. Clinical data were also recorded in order to evaluate tolerability.

\section{Results}

18 pts (15 F, 3 M) with a median age of 59,8 y (range 43-74) affected of PM from ovary (10), gastric (6), primary papilar (1) and endometrial (1) were treated. 11 of them were synchronic. 16 pts underwent previous chemotherapy and 2 pts radiotherapy. The mean $\mathrm{PCl}$ was 12,7 (2-39) and the grade of cytoreduction obtained: CCRO: 14, CCR1: 3 and CCR2: 1.

The dose administered to reach the target concentration (mean (SD)) was 736.76 (160.59) mg of oxaliplatin and 55.88 (18.04) $\mathrm{mg}$ of doxorubicin for a perfusion fluid volume of $3.61(0.86) \mathrm{L}$. At the beginning of the perfusion the median of the peritoneal concentration of oxaliplatin was $163.73(95.69-303.36) \mathrm{mg} / \mathrm{L}$ and 4.09 $(1.66-22.6) \mathrm{mg} / \mathrm{L}$ for doxorubicin. This concentration was reduced by $36.2 \%$ (oxaliplatin) and by $55.2 \%$ (doxorubicin) after 30 minutes of perfusion. Absorption of oxaliplatin and doxorubicin from peritoneum to blood was observed. Mean Cmax (SD) plasma concentrations of oxaliplatin were 7.01 (4.80) mg/L and 0.049 (0.022) $\mathrm{mg} / \mathrm{L}$ for doxorubicin.

6 pts presented grade III-IV complications in the postop (33\%) and 5 pt (27\%) required a surgical reintervention. 1 pt died due to sepsis and multiorgan failure. The mean hospital stay was 23 days (9-60).

5 pts needed hospital readmission due to intraabdominal abscess (2), intestinal obstruction (1) and malnutrion (1).

\section{Conclusion}

Mean serum concentrations detected were similar to those obtained after the IV administration of both agents at conventional doses used in clinical practice.

No relationship was observed between drugs exposure (Cmax) and postoperative complications.

The administration of oxaliplatin + doxorubicin based on concentration $(\mathrm{mg} / \mathrm{L})$ is feasible and does not increase the morbi-mortality in this subset of patients.

125

POSTOPERATIVE OXALIPLATIN IN THE BIOLOGICAL FLUIDS AFTER HEATED INTRAPERITONEAL CHEMOTHERAPY (HIPEC): A COMPARATIVE STUDY FOR PROCEDURES WITH AND WITHOUT LAVAGE

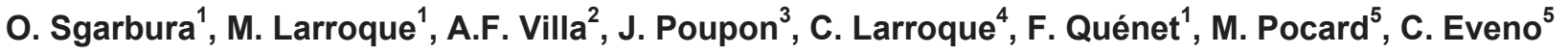

${ }^{1}$ Institute of Cancer Montpellier - Montpellier (France), ${ }^{2}$ Poison Center APHP - Paris (France), ${ }^{3}$ Toxicology laboratory, Lariboisière Hospital - Paris (France), ${ }^{4}$ Institut de Recherche en Cancerérologie Montpellier - Montpellier (France), ${ }^{5}$ Lariboisière Hospital - Paris (France)
} 


\section{Objectives}

Cytoreductive surgery and oxaliplatin-based heated intraperitoneal chemotherapy (HIPEC) are often performed in peritoneal carcinomatosis of digestive origins. While very strict safety measures are applied in the operative room, the presence of oxaliplatin in the biological fluids manipulated during the postoperative course is still unknown. The goal of this study was to dose oxaliplatin in the biological fluids of patients that received oxaliplatin-based HIPEC in two cohorts of patients: with and without postHIPEC perioperative lavage.

\section{Methods}

Biological liquids were sampled from four sources (abdominal drains, urinary catheters, nasogastric tubes, blood) during postoperative day (POD) 1 to 5 in two groups of patients: 10 patients who did not receive post HIPEC perioperative serum lavage and 11 patients who underwent lavage. The platinum levels were analyzed after centrifugation on ICP-mass spectrometry and were compared between the two groups for each sampling source and each POD.

\section{Results}

All patients were treated for colorectal and appendiceal peritoneal carcinomatosis and received an intraperitoneal dose of $460 \mathrm{mg} / \mathrm{m}^{2}$ of oxaliplatin. In both groups, a steep decrease of oxaliplatin levels is observed between POD 2 and 4 in all the biological fluids ( $p$-value $<10-3$ for all sampling sources). There is an average diminishment of the platinum levels of $52,3 \%$ in the lavage group versus $43,81 \%$ in the control group between POD2 and POD 4( $p=N S)$. There was no significant difference between the dose levels in the blood and urine but significantly higher levels of oxaliplatin were seen in the nasogastric tube and the pelvic drain in the lavage cohort.

\section{Conclusion}

Oxaliplatin levels in the drains diminish by almost $50 \%$ between POD2 and POD4 suggesting that drain removal is safer starting from POD4 although the threshold to increased exposure-related risk in healthy humans is currently not known. Furthermore, there is no impact of lavage on the postoperative presence of platins in the biological fluids and, therefore, no protective effect on postoperative caregivers.

126

EVALUATION OF NATURAL KILLER (NK) AND NATURAL KILLER T (NKT) CELL RECEPTORS IN OVARIAN CANCER

S. Mehta ${ }^{1}$, P. Kumar ${ }^{2}$, J.M. Pramanik², J. Anam ${ }^{1}$ P. Kammar ${ }^{3}$

${ }^{1}$ Saifee Hospital, Charni Road, Mumbai, Indiael, Mumbai, India - Mumbai (India), ${ }^{2}$ ICMR-Natioanl Institute for Research in Reproductive Health, JM Street, Parel, Mumbai, India - Mumbai (India), ${ }^{3}$ Global Hospital, Hyderabad - Hyderabad (India) 


\title{
Objectives
}

Several immune mechanisms have been reported to be associated with development of epithelial ovarian cancer (EOC). Natural killer (NK) and Natural Killer Tcells (NKT) are the prime immune effector cells in innate immunity,. However, their association with development of EOC and the mechanism of failure of recognition of EOC cells has not been adequately studied. The aim of this study was to evaluate the expression of NK and NKT cell receptors and their cognate ligand expression in EOC.

\section{Methods}

NK cell and NKT cells receptors were studied using Flow cytometer in 5 primary ovarian cancer cases and 4 healthy controls and corresponding cognate ligand expression was evaluated on the EOC tumour tissue samples obtained during surgery.

\section{Results}

NKG2D, CD161 and DNAM-1 is the dominant activating receptor expressed over NK and NKT cells. NKG2D, CD161 receptors have been found downregulated in ovarian cancer patients compared to healthy individual, in peripheral blood and tissue resident NK and NKT cells. CD161 expression is less affected on tissue resident NKT cell population. KIR3DL1, an inhibitory receptor is slightly upregulated on NK cell population in peripheral blood and NKp44 expression was increased. DNAM-1 is an adhesion receptor required for synapse formation between NK and cancer/ stressed cells. DNAM-1 expression is found downregulated in tissue resident NK and NKT cells, however not affected on NK and NKT cells in peripheral blood. NKp46 expression is downregulated over NK cell in peripheral blood, while no NKp46 expression was found on peripheral NKT cells. NKp46 expression is upregulated on tissue resident NKT cell population.

\section{Conclusion}

Expression of activating receptor is downregulated in ovarian cancer patients; alternatively expression of inhibitory receptor is upregulated on both the cell populations. Receptors like NKp44 and NKp46 found to be expressed in only tissue resident NK and NKT cell respectively. Expression of CD161 is less affected. Initial analysis revealed promising results on association of NK and NKT cells with ovarian cancer. Study in more number of cases may confirm these significant findings.

\section{COMPARISON OF EXPERIMENTAL MOUSE MODELS OF COLORECTAL PERITONEAL CARCINOMATOSIS}

\author{
A.T. Taibi ${ }^{1}$, M.L.P. Perrin ${ }^{2}$, L.C. Carr ${ }^{2}$, C.Y. Yardin ${ }^{3}$, P.L. Leveque ${ }^{2}$, D.C.A. Arnaud Cormos ${ }^{2}$, S.B.C. \\ Bardet $^{2}$, S.D.F. Durand Fontanier ${ }^{4}$ \\ ${ }^{1}$ Service de chirurgie digestive, CHU Limoges, Université de Limoges, XLIM, CNRS, UMR 7252 \\ Limoges - Limoges (France), ${ }^{2}$ université de Limoges, XLIM, CNRS, UMR 7252 Limoges - Limoges \\ (France), ${ }^{3}$ Service d'histologie, CHU Limoges, Université de Limoges, XLIM, CNRS, UMR 7252 \\ Limoges - Limoges (France), ${ }^{4}$ Service de chirurgie digestive, CHU Limoges, université de Limoges, \\ XLIM, CNRS, UMR 7252 Limoges - Limoges (France)
}




\section{Objectives}

For many decades, mice are used as model organisms to study human pathology thanks to the genetic and physiological similarities between both species. Different murine models of peritoneal carcinomatosis (PC) are described in the literature, based mainly on the graft methods: intraperitoneal injection (IP) of tumor cells, subcutaneous injection (SC), intravenous injection (IV), or intraperitoneal injection after peritoneum aggression by laparotomy $(\mathrm{L})$. Until today, none study have compared them under the scope of obtaining a limited PC. The aim of our work was indeed to compare 9 and 15 days after injection these four established models, with a constraint of peritoneal cancer index $(\mathrm{PCl})<10$.

\section{Methods}

40 female pathogen-free Balb-C mice, weighting 20-30 g, obtained from Charles River, were used in this study. The tumor cell line used was a moderately differentiated adenocarcinoma of the mice colon (luciferase-expressing murine CT-26 colon carcinoma cells, Narumi et al., 2012). The tumor cells were implanted by intraperitoneal injection (group IP), subcutaneous injection (group SC), intravenous group (group IV) and intraperitoneal injection after scraping of peritoneum with sterile cotton swab (group laparotomy). 10 mice from each group were studied daily between day 3 and 15 postgrafting, and sets of imaging data were acquired. First, mice were injected with a intraperitoneal solution of $25 \mathrm{mg} / \mathrm{kg}$ of XenoLight D-Luciferin (Perkin Elmer) under isoflurane anesthesia and, immediately after injection, imaged with a cooled charge-coupled device (CCD) camera system (IVIS ${ }^{2}$ Lumina System, Perkin Elmer). For quantitative analysis, a circular region of interest (ROI) was placed over the tumor, and the total signal (photons/s) in this ROI was determined using Image Analyst MKII software. At day 15, a laparotomy was performed and $\mathrm{PCl}$ score was calculated.

\section{Results}

The total signal (photons/s) was (0E), (2.21E + 05), (1.38E +05), $(4.95 \mathrm{E}+06)$ on day 9 and $(0 \mathrm{E}),(2.89 \mathrm{E}+$ $06),(6.75+06)$ and $(3.61 \mathrm{E}+07)$ on day 15 in groups IV, SC, IP and laparotomy respectively $(p<0.05)$. The $\mathrm{PCl}$ score was $0,1,2$ and 5 at Day 9 and $0,5,9$ and 23 at Day 15 in groups IV, SC, IP and laparotomy respectively $(p<0.05)$.

\section{Conclusion}

We successfully established a novel and fast animal model of limited colorectal peritoneal carcinomatosis thanks to a precise protocol of tumor cell injection. Among the four models, the intraperitoneal injection is the preferred model to obtain a limited PC at Day 15.

128

NANOSECOND PULSED ELECTRIC FIELD (NSPEF) TREATMENT FOR COLORECTAL PERITONEAL CARCINOMATOSIS

A.T. Taibi ${ }^{1}$, M.L.P. Perrin ${ }^{2}$, L.C. Carr ${ }^{2}$, P.L. Leveque ${ }^{2}$, C.Y. $\operatorname{Yardin}^{3}$, D. Arnaud-Cormos ${ }^{2}$, S.D.F. Durand Fontanier $^{4}$, S. Bardet ${ }^{2}$

${ }^{1}$ Service de Chirurgie Digestive, CHU Limoges, Université de Limoges, XLIM, CNRS, UMR 7252 Limoges - Limoges (France), ${ }^{2}$ Université de Limoges, XLIM, CNRS, UMR 7252 Limoges - Limoges (France), ${ }^{3}$ Service d'histologie, CHU Limoges, Université de Limoges, XLIM, CNRS, UMR 7252 Limoges - Limoges (France), ${ }^{4}$ Service de chirurgie digestive, CHU Limoges, Université de Limoges, XLIM, CNRS, UMR 7252 Limoges - Limoges (France) 


\section{Objectives}

Curative surgical treatment is only available to patients with resectable colorectal peritoneal carcinomatosis (PCc), leading to a lack of therapeutic alternatives for the numerous patients displaying unresectable PCc. High-intensity pulsed electric fields with nanosecond durations (3-300 ns; nsPEFs) have emerged as a promising tool for tumor ablation. The aim of this study is to evaluate the therapeutic effects and safety of nsPEFs used to treat PCc.

\section{Methods}

We have previously developed in vitro and in vivo nsPEF exposure setup composed of a nsPEF generator (FPG 10-1NM-T, 10ns pulses, amplitudes between $4.5 \mathrm{kV}$ and $10 \mathrm{kV}$, rise-times around $5 \mathrm{~ns}$ ), a digital phosphor oscilloscope, a high-voltage measurement device (tap-off), an electrode-based delivery system. CT26-luc1 cells were obtained from a BALB-c murine colon adenocarcinoma cell line expressing firefly luciferase (Narumi et al., 2012). They were analyzed in vitro by flow cytometry for their sensitivity to nsPEFs (survival, mitochondrial potential and permeabilization). In parallel, CT26-luc1 cells were grafted in immunocompetent BALB-C mice by intraperitoneal injection in order to develop a limited PCc before bioelectric treatment. Microscopic and histologic investigations were conducted in human and mice peritoneal tumoral tissue by multiphoton imaging in order to evaluate the tumor microenvironment.

\section{Results}

The dosimetry of delivery systems showed that the electric field in the central region between the electrodes had a good homogeneity, as based on 3D numerical simulations. The intensity of the electric field delivered in vitro in the electroporation cuvette was around $60 \mathrm{kV} / \mathrm{cm}$ and in vivo was between $30-50 \mathrm{kV} / \mathrm{cm}$. Multiphoton microscopy showed tumoral fibrosis by second harmonic generation (SHG) and infiltrated CT26luc1 tumoral cells, in comparison with non-pathologic peritoneum. In vitro data are also consistent with our published results on 5 tumoral cell lines (Soueid et al., 2017), we observed a strong dose-dependent effect of nsPEF on cell viability.

\section{Conclusion}

We have demonstrated that CT26-luc1 cells are sensitive to 10 ns nsPEF. We have also developed a suitable murine model for studying PCc after bioelectric treatments. Current experiments are done by combining fluorescent and bioluminescent reporters to measure tumour regression after nsPEF treatments. Our future data will help to clarify the signaling pathways activated in vivo in the hours and days following nsPEF application. 